\title{
LA INVESTIGACIÓN FORMATIVA EN LA UNIVERSIDAD
}

\section{NACIONAL ALTIPLANO DE PUNO}

\author{
Cesar Machaca Escobar*
}

\section{INFORMACIÓN DEL ARTÍCULO}

Art. Recibido: 04/08/2020

Art. Aceptado: 04/08/2020

Art. Publicado: $10 / 08 / 2020$ 


\title{
RESUMEN:
}

La humanidad está sujeto al desarrollo de la ciencia y la tecnología, en la actualidad estas se retroalimentan con los saberes, y de los conocimientos. En este sentido, la reciente implementación del currículo por competencias la Universidad Nacional del Altiplano, prioriza la formación académica mediante los componentes curriculares que tienen como finalidad la investigación formativa respecto al estado de cosas, sobre todo la discusión y la conclusión, a través de la acopio y sistematización de información y resultados en un contexto, el cual permite la familiarización de los estudiantes de pregrado.

Para ello, la labor de la universidad es investigar y como tal constituye el epicentro de la construcción del conocimiento con un tratamiento epistemológico sujeto a la dinamicidad y adaptación a la crisis de un modelo, y así eclosione otro. La sociedad académica en el marco de la construcción del conocimiento contextual en nuestra primera casa superior de estudios como parte de la comunidad científica. Son retos para impulsar el cambio de concepción en la educación universitaria, de tal modo es una época de conversión del encasillamiento en escritorio e instrucción mecanizada por la internalización mediante el contacto con la realidad en las diversas áreas del saber.

Palabras Claves: Competencia, investigación científica, investigación formativa, investigación, método científico.

\section{FORMATIVE RESEARCH AT THE ALTIPLANO DE PUNO NATIONAL UNIVERSITY}

\begin{abstract}
:
Humanity is subject to the development of science and technology, currently these are fed back with knowledge, and knowledge. In this sense, the recent implementation of the competency-based curriculum at the National University of the Altiplano prioritizes academic training through curricular components that are aimed at formative research regarding the state of affairs, especially discussion and conclusion, through the collection and systematization of information and results in a context, which allows the familiarization of undergraduate students.

For this, the university's job is to investigate and as such constitutes the epicenter of the construction of knowledge with an epistemological treatment subject to the dynamics and adaptation to the crisis of one model, and thus hatch another. The academic society within the framework of the construction of contextual knowledge in our first higher house of studies as part of the scientific community. They are challenges to promote the change of conception in university education, in this way it is a time of conversion of the typecasting in desk and instruction mechanized by internalization through contact with reality in the different areas of knowledge
\end{abstract}

Keywords: Competence, formative research, research, scientific method, scientific research. 


\section{Introducción}

A diario el hombre esta en la necesidad de indagar, pues para ello requiere adiestrar la competencia que es parte de la educación y la psicologia, capacidad inherente del hombre. La investigación en la Alma Mater, ha sido fruto de la reforma polémica denominado Ley Mora, con la dación de la Ley Universitaria 30222 a los ocho días del mes de julio del dos mil catorce, en la capital de la Republica, en el artículo siete punto dos establece la: "Investigación, por ser una función primordial y obligatoria en producir conocimiento", en concordancia al artículo 48.

Es una política institucional sujeta a la producción del conocimiento, cuya responsabilidad no solo recae en los directivos, ni el vicerrectorado de investigación, sino es el interés común de los miembros de la comunidad universitaria con la cooperación de decanos, coordinadores de investigación, docentes, administrativos y estudiantes para la implementación de la investigación formativa.

Para la concreción de esta reforma mediante la política institucional se regentó la: "Superintendencia Nacional de Universidades" (SUNEDU), además de su adscripción al artículo 12 como: "Parte de la Cartera de Educación y es un órgano fiscalizador", además es: "El órgano rector del licenciamiento", tal como reza el artículo 13, que exige el: "Licenciamiento" en el artículo 30. En este sentido, una vez que cumplan los criterios y estándares reciben beneficios e incentivos como la propulsión de los Institutos de Investigación.

Para realizar estas aspiraciones y responder al medio se vale del currículo que moviliza la gestión de conocimiento, particularmente el Proyecto Educativo Universitario (PEU-2013). Propicia la formación académica con congruencia mediante el currículo por competencias y la investigación formativa a través de los componentes curriculares que están comprendidos en el diseño curricular. Razón por la cual debe responder a la demanda de cada escuela profesional, al contexto regional y nacional, ya que legalmente obedece al artículo 40: "Ser renovada y actualizada cada tres años, sin soslayar los cambios de la ciencia y tecnología".

Atwell (2009), atribuye la génesis de la competencia a la sociología al margen de su definición dificultosa, puntualiza a la sociología industrial por la remuneración de los obreros, empleándose en las áreas de sociales e ingenieras. De algún modo, con Manríquez (2012), tiene alguna similitud sobre el trabajo. No obstante, es un antecedente previo al currículo universitario que se diseñan en los planes de estudios que conducen al desarrollo de habilidades. Asimismo, para Vázquez (2003), esta precedido por el lenguaje la competencia y es el desempeño. Manríquez (2012), relaciona con la parte física y mental, al igual que Vázquez (2003), quien segmenta en cognitivo y rendimiento.

Por su parte, el currículo por competencias según Vásquez (2003), es un proceso, al cual Sánchez y Daysi (2012), manifiestan que es la causa colectiva sujeto a los requerimientos de la diversidad y la realidad. Al respecto, Vásquez (2008), señala que es una actitud y un medio para la internalización de las competencias. Por último, para Sánchez y Daisy (2007), no existe subordinación sino la interdisciplinaridad. Sin embargo, Manríquez (2012), cuestiona a la globalización que genera crisis y Márquez (2012), reconoce el avasallamiento de las Tecnologías de Información y Comunicación (TIC), que de laguna manera inducen al pragmatismo, dejando de lado la acuciosidad de la información que ofrecen estos medios.

La investigación para Zermeño (2001), es un proceso objetivo. En cambio, para Niño es una habilidad parte del pensamiento y coincide con Zermeño (2001), quien 
Journal homepage: http://revistas.unap.edu.pe/rd/ E-mail: revistaderecho@unap.edu.pe

atribuye a las operaciones del pensamiento. La investigación formativa según Zapata (2010), es una forma de validar y corroborar. Mientras, para Naupas, Mejía, Novoa, \& Villagómez (2014), sus orígenes está en Colombia y persigue la cultura investigativa de los universitarios, y en el Perú, se funda el Sistema Nacional de Evaluación Acreditación y Certificación de la Calidad Educativa (SINEACE-2006) y la Comisión Nacional de Evaluación y Acreditación Universitaria (CONEAU-2008).

Para Restrepo (2003), es exploratoria-diagnóstico para dar las razones sobre el estado de cosas, empelando el método investigativo. Chiroque (2018), afirma que es una estrategia de disquisición prioritariamente individual, que según Sánchez y Daysi (2007), sugiere una labor grupal. La investigación científica para Restrepo (2003), es un conocimiento local y nuevo, además para Sánchez y Daysi (2007), prevalece el contexto y a partir de ello, se afianza el uso del método científico y Ortiz (2004), considera como una forma de sistematización.

\subsection{Enunciado}

En merito a la normativa vertida sobre la competencia en el desarrollo de la investigación formativa, esta requiere ser problematizada y no solo concebir como un indicador o estándar y mero cliché. La misma señalada por el ordenamiento jurídico y normas conexas que encaminan hacia la investigación, en consecuencia surge la incógnita macro como: ¿Cuáles son las acciones que realiza el vicerrectorado académico de la casa superior?, y a nivel micro así como: ¿Cuál es la política institucional para los estudiantes de pregrado la investigación? ¿Cuáles son los presupuestos teóricos para su concreción?

\subsection{Marco teórico conceptual}

\subsubsection{Competencia y competencias}

La competencia, conceptualmente arguye Attewell (2019), es: "un concepto lógico, complejo y ambiguo. Es de importancia en la sociología, objeto de estudio respecto a la desigualdad salarial y de las críticas al capitalismo que pauperiza el trabajo en tópicos de equidad laboral, proviene de la sociología industrial" (p. 21), además Attewell (2019), señala que el concepto competencia: "es importante en economía, psicología, educación, inteligencia artificial informática, y en el área conocida como recursos humanos o ergonomía" (p.22).

Para Manríquez (2012), el concepto de competencia es: “común en el contexto laboral, a finales de la última década del siglo pasado se ha incorporado en currículo universitario como parte de los modelos curriculares que cronológicamente data de los años 90 sufriendo una metamorfosis en sus denominaciones" (p. 308). Asimismo, destaca que la formación por competencias, está comprendido por: "planes de estudio basados en el enfoque por competencias, propuestas educativas por competencias; que buscan una formación académica alternativa" (Manríquez, 2012, p. 368).

Para Manríquez (2012), el concepto de competencia comprende la: "habilidad como capacidad de hacer bien y emplear la habilidad mental como la física, así como parte de ser destreza o habilidad física". Esta última noción, detalla diacrónicamente es parte de la Ilustración, así como aproxima Manríquez (2012), la competencia incursiona en el: "predominio físico de la capacidad, a nivel del léxico; el arcaísmo evidencia como astuto, diestro, don y hábil, en verdad es complejo" (p. 308). Por su parte Manríquez (2012) finaliza arguyendo que: "existe una complementación y simbiosis entre lo mental y físico de la competencia como parte del discurso sociológico que destaca la pericia, empero la parte física es desapercibido ocasionando un vacío y ambigüedad" (p. 368). 


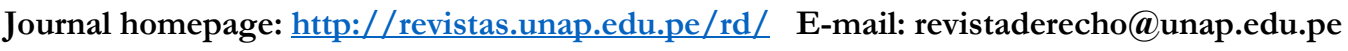

Por tanto, la competencia es: "la habilidad para hacer una acción, al mismo tiempo desprende la bondad de habilidad afluente. De tal forma, es sinónimo de destreza, y recuerda imágenes de domino, pericia, maestría, habilidad y excelencia" (Manríquez, 2012, p. 368).

Según Vázquez (2003), realiza discernimiento entre competencia y competencias. La competencia es superficial que: "proviene de otras disciplinas científicas y del lenguaje corriente". Conceptualmente, detalla que la competencia es: "el desempeño eficaz respecto a una situación nueva". Arguye que el desarrollo se: "consigue mediante las capacidades del individuo y su interacción". En síntesis, es la: "habilidad desarrollada y practicada, que se materializa en el saber hacer" (p. 56).

\subsubsection{Modelo curricular}

El modelo curricular en la universidad debe estar a la par con la realidad, es decir, obedecer a las demandas mediante un programa curricular que responda a la necesidad local, regional y nacional, de tal forma pueda adecuarse, así como Sánchez y Daisy (2007), asumen la postura de: "la construcción de conocimientos, habilidades, destrezas y actitudes" (p. 2), dichas afirmaciones exigen y buscan alcanzar el saber hacer al igual que Vásquez (2008) que propone dicho ideal, y es así que Sánchez y Daisy (2007), asumen que son: "adquiridos y desarrollados para un adecuado desempeño grupal sin limitarse en el conocimiento" (p. 2).

Es por ello, oportuno concebir e impulsar un modelo curricular contextual y funcional, así como Sánchez y Daisy (2007), propone: "un modelo curricular plural que forme ciudadanía, para comprender la alteridad", asimismo señala que: "no existen ciencias que subordinen al resto, de lo contrario sea complementario y sea un mecanismo interdisciplinario y transdisciplinario, superando prejuicios", y así lo: "cotidiano pueda ser una manera de construir o legitimar saberes contextuales" (p. 2).

Mientras, que las competencias son más profundas, porque exige que: "las capacidades se desarrollen mediante la interrelación entre los saberes y los ejes dinamizadores", esto para Vázquez (2003) es un mecanismo transitorio, procesal e intermediario entre la adquisición y desarrollo como parte de la internalización que para este autor se: "constituyen en conocimientos, habilidades, actitudes y destrezas" (p. 56).

\subsubsection{Las Tecnologías de la Información y Comunicación}

Las Tecnologías de la Información y Comunicación es la causa de las profundas crisis según Manríquez (2012), que el cual se ocasiona en el: "contexto de la globalización y las nuevas tecnologías de la información que evolucionan incesantemente" (p. 368), denominada como época de la cibernética que el cual conduce a los cambios económicos y culturales principalmente, ya que solo beneficia a los monopolios de aquellos países que proveen equipos, paquetes e inculcan la falsa necesidad del uso excesivo de las Tecnologías de la Información y Comunicación (TIC), accediendo a páginas con menor rigurosidad y parcialmente generan desigualdades en la población con restricción económica, por otro lado es latente el descuidado de los textos clásicos que contienen teorías como consecuencia surge la actitud pragmática en los estudiantes y ello conlleva a la repetición de conocimientos mas no el análisis.

Según Márquez (2012), estos efectos son parte del: "Vertiginoso avance científico en un marco socioeconómico neoliberal-globalizador y sustentado en el uso de las potentes y versátiles de las TICs" (p. 3). Pese a ello, la esperanza de muchos ciudadanos está en la universidad como protagonistas para propulsar alternativas de 


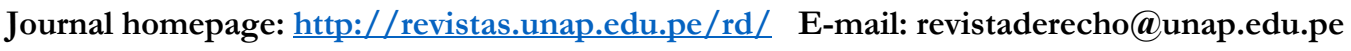

desarrollo cultural, económico, ambiental y social mediante saberes y el conocimiento adecuadamente administrados y usados de fuentes fiables.

\subsubsection{Investigación}

La investigación para Zermeño (2001) es un: "Proceso, objetivo, sistemático, reflexivo y ordenado que tiene como finalidad responder una pregunta y elaborar una solución" (p.1.). Para ello, es necesario conocer la acción misma que está constituida por la actividad que realiza el hombre frente a un enunciado, entonces esto demanda la búsqueda de solución. En tanto, investigar es: "inherente a la vida del ser humano", a su vez es el empleo de la operación del pensamiento que el tratadista enfatiza: "es inseparable de la actividad cognoscitiva del sujeto" (Niño, 2011, pp.24-25).

Para Hernández (2003), inquirir es: "seguir los vestigios, huellas, descifrar los rastros. Es próximo a la búsqueda de una causa, y también para nombrar los procesos sistemáticos de producción de conocimiento académico" (p. 184). Para Ortiz (2004), la indagación consiste en: "el procedimiento reflexivo, sistemático, controlado y crítico, que permite descubrir nuevos hechos o datos, relaciones o leyes, en cualquier campo del conocimiento humano" (p. 90).

\subsubsection{Investigación formativa}

Surge más con un sentido exploratorio, ya que esta metodología no tiene el propósito según Zapata (2010), de: “aceptar a pie juntillas o rechazar contundentemente el objeto de investigación, todo lo contrario es para valorar la eficacia y alcanzar mejoras en función de ejecuciones progresivas y controladas" (p.1.).

La investigación formativa en el Perú, según Naupas, Mejía, Novoa, \& Villagómez (2014) es: "un modelo reciente que tiene como finalidad alcanzar la acreditación de las escuelas profesionales impulsado por el SINEACE y la CONEAU (2008)". Al mismo tiempo, sostienen que sus orígenes está en el país de: "Colombia como parte de la reforma universitaria donde incorporaron la investigación formativa a través de la Ley 30 de 1992, creando el Consejo Nacional de Acreditación (CNA)". En efecto, Restrepo (2003), manifiesta que fue para impulsar: "la cultura investigativa" a partir de la: "exploración y descripción" (p.106).

La investigación formativa tiene relevancia en el aprendizaje por descubrimiento tal como Restrepo (2003) afirma que su: "presencia es consustancial, ya que permite descubrir y construir conocimiento". Plantea tres trópicos próximos como son: "la exploración; comprende el diagnóstico y documentación, formación; familiarización; en la actividad y la trasformación; arriba a un plano práctico. Por último, señala que se emplea el método y se practica en los trabajos de grado con el apoyo de un asesor que cumpla con su función exigente y rigurosa para que tenga un hilo conductor y argumento, y la otra forma es con la inducción del profesor investigador" (pp. 197200).

\subsubsection{EI método investigativo}

Al propósito, con un desarrollo más puntual y contextual Chiroque (2018), sostiene que es propicio el uso del: "método investigativo en cada uno de los componentes curriculares a implementarse como parte del objeto de estudio", a través del tratamiento de categorías y estrategias como son el: "problema, el trabajo individual o grupal, exposición, juicio crítico, conclusiones y señalamiento dialogado" (pp. 55-56).

\subsubsection{Investigación científica}




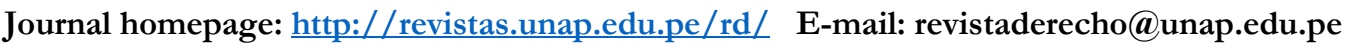

Restrepo (2003), contraria señalando que: "la investigación en la universidad no puede circunscribirse a la modalidad formativa", sino que asume: "la investigación formativa produce conocimiento local, subjetivamente nuevo" (p. 201), finaliza afirmando que los: "criterios para adelantar y juzgar la investigación científica rigurosa esperada de la universidad de acuerdo con la misión que ella ha establecido y que la sociedad demanda. A ella pueden vincularse también estudiantes en calidad de auxiliares, participantes y coinvestigadores" (p.202).

\subsubsection{Método científico}

El método científico según Ortiz (2004), comprende las: "teorías, hipótesis, variables, indicadores y el contexto", asimismo se caracteriza por ser: "fáctico, va más allá de los hechos, se vale de la observación, se ajusta a las conclusiones, sus formulaciones son de tipo general y objetivo" (p. 107).

\subsection{Objetivos}

El propósito de este escrito académico busca analizar las acciones que realiza el vicerrectorado académico, así como determinar la política institucional con los estudiantes de pregrado en la investigación y detallar los presupuestos teóricos para su concreción.

\section{Metodología}

Para el desarrollo de este artículo científico en el plano metodológico, el enfoque que se usó fue el cualitativo, para ello se hizo mediante la técnica del análisis documental.

\section{Resultado}

Respecto al resultado del origen de la competencia tiene cercanía con la psicología industrial, su complejidad, la procedencia del ámbito laboral que más adelante es parte del currículo universitario. Asimismo, la competencia por su carácter de bidimensionalidad, a ello se suma las demandas a las que debe estar sujeta el currículo de forma plural sin prejuicios, sin embargo, las TICs por el uso excesivo causan efectos negativos como el uso pragmático de la información de páginas de menor rigurosidad.

La investigación es parte de la actividad humana que realiza el hombre, en este contexto es puntual destacar la investigación formativa como adiestramiento y familiarización en el uso del método investigativo más certero que es el trabajo grupal para ello es necesario aplicar la investigación científica para indagar conocimientos locales, usando adecuadamente el método científico, en seguida exponemos las proximidades y diferencias de cada autor que aborda respecto a la competencia y a la investigación.

En cuanto a su génesis, la competencia según Vázquez (2003), atribuye a otras disciplinas del lenguaje, en este sentido Attewell (2009), cataloga como complejo y asume que procede de la sociología industrial, rama especializada que cuestiona las brechas salariales al modelo capitalista que es producto de los rezagos del feudalismo que genero las desigualdades y la explotación del hombre, sitúa como interdisciplinaria a la economía, psicología, educación, inteligencia artificial informática, y a los recursos humanos o ergonomía, este postulado coincide con Manríquez (2012), que reitera su origen en el contexto laboral, puntualiza que el currículo es parte de los 90 denominado como plan de estudio.

Sobre el concepto mismo, para Manríquez (2012), la competencia es la habilidad de realizar una acción de forma acertada, de forma similar Vázquez (2003), detalla que 
Journal homepage: http://revistas.unap.edu.pe/rd/ E-mail: revistaderecho@unap.edu.pe

es el desempeño eficaz que consiste en el saber hacer. Teóricamente Manríquez (2012), rebate respecto a su connotación semántica por tener una cualidad o naturaleza profunda sobre la definición de Vázquez (2003), al concebir que la competencia es tipo bidimensional porque el sujeto usa la parte mental y física complementariamente y esta última categoría es entendida como astucia, siendo desapercibido.

En otra perspectiva a nivel del currículo las competencias según Vásquez (2003), es un proceso y para Sánchez y Daysi (2012), es un desempeño grupal, en cuanto a las competencias propiamente dicho Sánchez y Daisy (2007), sugiere un nuevo modelo curricular universitario que engarce con las necesidades, además impulsa el enfoque curricular por competencias internalizados como resultado del trabajo en equipo, no solo la mecanización de los contenidos supeditado a la actitud, habilidad y destreza.

Asimismo, recomienda la pluralidad del currículo para formar ciudadanía empática frente a la diversidad de tal forma promueve la construcción del conocimiento contextual y frente a la competencias que se quedó en el saber hacer, Sánchez y Daisy en ese sentido se preocupan por el saber contextual, sin embargo tiene una proximidad a nivel de la definición de manera superficial que es una similitud de Vásquez (2008), en términos de actitud y destreza, con la particularidad que destaca como proceso e intermedio entre la adquisición y el desarrollo.

Por último, Sánchez y Daisy (2007), reitera que deben ser abordados adecuadamente el conocimiento, ya que no existe superioridad ni inferioridad del conocimiento. Por el contrario promueve el mecanismo interdisciplinario y transdisciplinario, para evitar verticalidades absolutas que caen en dogmas constituyéndose en prejuicios.

Sin embargo, Manríquez (2012), detalla que la globalización y las nuevas tecnologías de la información ocasionaron cambios incontrolables para Márquez (2012), la causa es el vertiginoso avance científico que proviene del marco socioeconómico neoliberal-globalizador que desapercibe el desarrollo de las competencias hace lo contrario en generar actitudes pragmáticas respecto al uso de la información de poca rigurosidad.

En esta segunda parte, la investigación para Zermeño (2001), es un proceso y objetivo porque que inicia con el enunciado seguido de la hipótesis, es decir, se pretende ubicar al objeto por encontrar o probar la afirmación tentativa en forma de respuesta del cual se sospecha, de tal forma que se parte de un problema y se postuló la solución denominado hipótesis, este autor consecuentemente complementa que es sistemática, reflexiva y ordenada.

Mientras que para Niño (2011), concluye definiendo como una habilidad y cualidad inherente al hombre por ser inseparable a la parte cognitiva, esta última categoría es próxima al pensamiento tal como afirma Zermeño (2001), que es parte de las operaciones del pensamiento.

La investigación formativa para Zapata (2010), no es el rechazo absoluto, sino es parte de la corroboración de su eficacia. Para Naupas, Mejía, Novoa, \& Villagómez (2014), es un modelo emergente de Latinoamérica, específicamente que surge en Colombia (1992), con el propósito de generar la cultura investigativa en el sistema universitario como parte de la reforma universitaria.

En el Perú, después de dieciséis años se institucionaliza el Sistema Nacional de Evaluación Acreditación y Certificación de la Calidad Educativa (SINEACE) y la Comisión Nacional de Evaluación y Acreditación Universitaria (CONEAU-2008) incorporaron la acreditación de las escuelas profesionales.

Mientras, que para Restrepo (2003), es la fase exploratoria donde se recure al diagnóstico, la documentación, a partir de ello desarrollar un argumento fluido y 


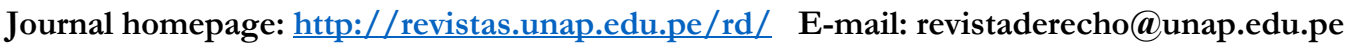

contunde. En cuanto, al método investigativo para Chiroque (2018), es el uso de estrategias para el desarrollo de los componentes curriculares identificando problemas para una discusión individual o grupal y así arribar a la conclusión. Es conveniente hacer uso del trabajo individual y alternativamente el grupo, trasuntando al currículo por competencias, sin embargo cuestiona Sánchez y Daysi (2007), prioriza el tratamiento grupal, y puntualiza que es asidero para la adquisición de competencias.

La investigación científica según Restrepo (2003), es parte del conocimiento local nuevo y que la sociedad demanda, y esto a nivel del currículo se recoja para Sánchez y Daysi (2007), es propicio superar perjuicios, ya que el contexto es un criterio para la actualización del plan de estudios.

Asimismo, la investigación científica, busca que los educandos indaguen los conocimientos locales, de tal manera se han inducidos para abordar problemáticas de mayor magnitud, y así participen como auxiliares y coinvestigadores en los trabajos de investigación.

El método científico para Ortiz (2004), las teorías son prioritarias que está comprendida en textos clásicos, a partir de ello reorientar el enunciado en forma general y específica para cada investigación en particular, sin desapercibir el contexto e incide que los estudios sean objetivos. Entonces, la investigación formativa sea empleado adecuadamente para arribar a una conclusión puntual y específica.

\section{Conclusiones}

El Vicerrectorado Académico promueve la investigación, tal como exige la Superintendencia Nacional de Universidades, sin embargo por la naturaleza del tiempo aún no se visibiliza por ser parte de un trabajo a mediano y a largo plazo, pero en el corto periodo existen acciones para afianzar el currículo por competencias y la investigación formativa mediante el Proyecto Educativo Universitario y el manual para el desarrollo de competencias en investigación formativa, instrumentos que tienen relevancia complementaría para la concreción de las aspiraciones como centro superior que atiende a la problemática regional de Puno, parte de la agenda con la cooperación de cada una de las escuelas profesionales.

La implementación como parte de la política institucional es producto del Proyecto Educativo Universitario, que en el cual establecieron las competencias que en la actualidad es parte del currículo por competencias exigido por la legislación vigente de la Superintendencia Nacional de Universidades, la competencia es antecedida y se caracteriza por el saber hacer como producto, mientras que las competencias es una categoría seminal que comprende etapas o fases que finalmente es el intermedio y parte del proceso de internalización que recae entre la adquisición y el desempeño, que en los noventa pasa a ser parte del currículo universitario denominado como plan de estudio. Los componentes curriculares son actualizados cada tres años como ideales los planes de estudio que responden a la demanda de la sociedad, según el contexto, la ciencia y la tecnología que afianzan las destrezas a partir del tratamiento de la problemática local mediante la investigación formativa para abordar trabajos de pregrado, indirectamente, directamente y en el futuro.

Los presupuestos y recursos teóricas han sido sistematizados de forma oportuna con la publicación del manual para el desarrollo de competencias en investigación formativa de Sigfredo Chiroque Chunga (2018), editado por la Universidad Nacional del Altiplano-Puno con edición de Tarea Asociación Grafica Educativa de 226 páginas con registro ISBN 978-612-4295-68-3, el cual reúne las categorías básicas de la investigación que a nivel bibliográfico adolecía a investigación formativa y es un material de consulta para los estudiantes de pregrado respecto a la ciencia, escritura, 


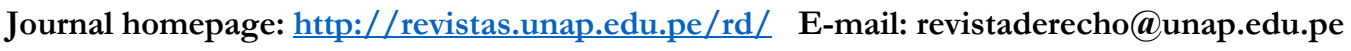

artículos, monografías, ensayos, informes y normas de redacción como tópicos que contribuyen e incentivan en la consolidación de la investigación formativa en la región sur del país.

\section{Consideraciones finales}

Las consideraciones finales convenientes es la inducción y la socialización hacía de la investigación por parte de Vicerrectorado Académico, a los miembros de la comunidad universitaria para así alcanzar con los fines de la universidad.

La implementación de la política institucional a través del currículo por competencias y la investigación formativa, exige evaluación, seguimiento y retroalimentación sobre la funcionalidad y discernimiento tanto del contexto occidental y amazónico andino regional para posteriores actualizaciones de expertos y los agentes directamente involucrados.

Los presupuestos y recursos teóricos deben ser producidos e ilustrados por autoridades académicas en la investigación tanto de la región y del país, en cada una de las ramas del saber para dar marcha adecuada de la investigación formativa mediante las ediciones futuras de manuales por áreas.

\section{Referencias bibliográficas}

Attewell, P. (2009). ¿Qué es una competencia? Pedagogía Social,Vol.16, No. 2, pp. 2143.

Chiroque, S. (2018). Manual para el desarrollo de competencias en investigación formativa. Puno: Tarea Asociación Grafica Educativa.

Hernández, C. A. (2003). Investigación e investigación formativa. Nómadas, No. 18, pp. 183-193.

Manríquez, L. (2012). ¿Evaluación en competencias? Estudios Pedagógicos, Vol. 38, No.1.pp. 367-380.

Marqués, P.R. (2013). Impacto de las TIC en la educación: funciones y limitaciones. Cuadernos de desarrollo aplicados a las TIC, Vol. 2, No.1, pp. 1-15.

Ministerio de Eduacion del Perú (2014). Ley universitaria 30220 Recuperado de www.reformauniversitaria.pe

Niño, V. M. (2011). Metodología de la investigación: Diseño y ejecución. Colombia: Editorial Ediciones U.

Ñaupas, H.; Mejía, E.; Novoa, E. \& Villagómez, A. (2014). Metodología de la investigación. $4^{\circ}$ ed. Bogotá: Editorial U.

Ortiz, F. G. (2010). Diccionario de metodología de la investigación científica. México: Editorial Limusa.

Restrepo, B. (2003). Investigación formativa e investigación productiva de conocimiento en la universidad. Nómadas, No 18, 195-202.

Universidad Nacional del Altiplano.(2013). Proyecto Educativo Universitario: Hacia una educación universitaria socicritica cognitiva. Puno: Editorial El Altiplano.

Vázquez, W. (2003). Diccionario pedagógico. Lima: Editorial San Marcos.

Zapata, M. (2010). La investigación formativa y la investigación basada en el diseño: Dos perspectivas de alcance. Educación a Distancia, No. 22, pp.1-3.

Zermeño, S.(2011). El ABC de investigar. León: Editorial Pearson. 